\title{
Diabetes Medication Use in Association with Survival among Patients of Breast, Colorectal, Lung, or Gastric Cancer
}

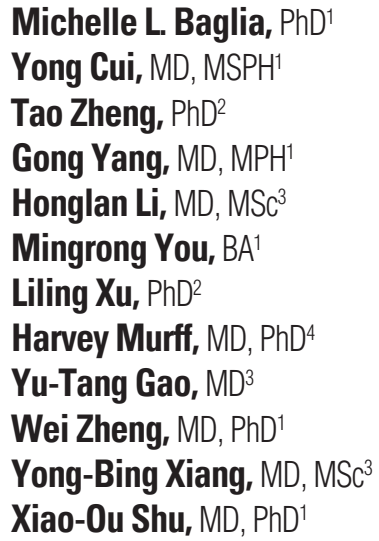

${ }^{1}$ Division of Epidemiology, Department of Medicine, Vanderbilt-Ingram Cancer Center, Vanderbilt University Medical Center, Nashville, TN, USA, ${ }^{2}$ Tongren Hospital, Shanghai Jiao Tong University, Shanghai, ${ }^{3}$ Shanghai Cancer Institute, Shanghai Jiao Tong University, Shanghai, China,

${ }^{4}$ Division of General Internal Medicine, Department of Medicine,

Vanderbilt University, Nashville, TN, USA

\section{Purpose}

Studies suggest that regular use of metformin may decrease cancer mortality. We investigated the association between diabetes medication use and cancer survival.

\section{Materials and Methods}

The current study includes 633 breast, 890 colorectal, 824 lung, and 543 gastric cancer cases identified from participants of two population-based cohort studies in Shanghai. Information on diabetes medication use was obtained by linking to electronic medical records. The associations between diabetes medication use (metformin, sulfonylureas, and insulin) and overall and cancer-specific survival were evaluated using time-dependent Cox proportional hazards models.

\section{Results}

After adjustment for clinical characteristics and treatment factors, use of metformin was associated with better overall survival among colorectal cancer patients (hazards ratio [HR], 0.55; $95 \%$ confidence interval [Cl], 0.34 to 0.88 ) and for all four types of cancer combined (HR, $0.75 ; 95 \% \mathrm{Cl}, 0.57$ to 0.98). Ever use of insulin was associated with worse survival for all cancer types combined (HR, 1.89; $95 \% \mathrm{Cl}, 1.57$ to 2.29 ) and for the four cancer types individually. Similar associations were seen for diabetic patients. Sulfonylureas use was associated with worse overall survival for breast or gastric cancer (HR, 2.87; 95\% Cl, 1.22 to 6.80 and $\mathrm{HR}, 2.05 ; 95 \% \mathrm{Cl}, 1.09$ to 3.84 , respectively) among diabetic patients. Similar association patterns were observed between diabetes medication use and cancer-specific survival.

\section{Conclusion}

Metformin was associated with improved survival among colorectal cancer cases, while insulin use was associated with worse survival among patients of four major cancers. Further investigation on the topic is needed given the potential translational impact of these findings.

\author{
Correspondence: Xiao-Ou Shu, MD, PhD \\ Division of Epidemiology, Department of \\ Medicine, Vanderbilt Epidemiology Center, \\ Vanderbilt University Medical Center, \\ 2525 West End Avenue, Suite 600 (IMPH), \\ Nashville, TN 37203-1738, USA \\ Tel: 1-615-936-0713 \\ Fax: 1-615-936-8291 \\ E-mail: xiao-ou.shu@vanderbilt.edu \\ Received December 14, 2017 \\ Accepted June 25, 2018 \\ Published Online July 2, 2018
}




\section{Introduction}

Diabetes is associated with an increased risk of cancers, as well as overall/cancer mortality [1]. Recent studies have shown that metformin and other diabetes medications may reduce risk or mortality for some cancers [2,3], although findings are inconsistent [4].

A recent meta-analysis reported that metformin use was associated with reduced overall cancer mortality, and liver cancer and breast cancer mortality individually [4]. Overall cancer mortality was evaluated in four studies, all of which observed decreased mortality among metformin users compared to non-users [5-8], although one study's findings did not reach statistical significance [5]. One study evaluated mortality by cancer type and found that metformin use was associated with decreased mortality from lung cancer, but observed no association with breast or colorectal cancer [8].

Fewer studies have evaluated cancer survival, rather than mortality, and its association with metformin. For breast cancer survival, one study observed improved survival among stage $\geq 2$, HER $2+$ breast cancer patients [9], while another study found no association in survival among triple-negative breast cancer patients [10]. One previous study in colorectal cancer patients found significantly increased survival among metformin users [11]. Other recent studies, not included in the meta-analysis, were identified. One study among lung cancer patients found no significant survival benefit for metformin users when compared to either non-diabetics or diabetic non-users [12]. A study among diabetic patients with non-small cell lung cancer found significantly reduced mortality among patients with longer duration of metformin use, particularly those who began taking metformin prior to lung cancer diagnosis [13]. A study among colorectal cancer patients with and without diabetes found that metformin use was associated with better survival compared to diabetics taking other diabetes medication [14], while another study found that metformin use among diabetics was associated with better survival compared to diabetic patients taking any or no other diabetes medications [15]. A study evaluating metformin use and gastric cancer reported that the increased cumulative duration of metformin use decreased the recurrence, all-cause mortality, and cancer-specific mortality rates among gastric cancer patients with diabetes who underwent gastrectomy [16], while another study suggested that metformin use might improve overall mortality, but not cancerspecific survival [17].

Fewer studies have been published evaluating the association between cancer mortality/survival and other diabetes medications, such as sulfonylureas or insulin. One study found that sulfonylureas or insulin monotherapy in the three months prior to cancer diagnosis was associated with higher overall cancer mortality compared to non-diabetics [8]. Another study found the cumulative dose exposure to insulin was associated with lower cancer mortality among diabetic patients taking insulin [18]. Metformin users have been shown to have lower mortality rates compared to sulfonylureas or insulin users [19].

To date, epidemiologic studies on the association between diabetes medications and cancer survival are limited and the findings inconsistent. Furthermore, there is a serious concern that immortal time bias (in epidemiology, immortal time refers to a period of cohort follow-up or observation time, during which death cannot occur) may have influenced many studies that examined medication exposure, including metformin use, and cancer survival [20-22]. We report here a study of 2,890 incident cancer cases (633 breast, 890 colorectal, 824 lung, and 543 gastric) identified from participants of the Shanghai Men's Health Study (SMHS) and the Shanghai Women's Health Study (SWHS). Using information obtained via electronic medical records (EMR), we examined the associations between metformin, sulfonylureas, and insulin use and survival from breast, colorectal, lung, and gastric cancers with a time-dependent analytic approach.

\section{Materials and Methods}

\section{Ascertainment of cancer cases}

Incident cases of breast, lung, colorectal, or gastric cancer were identified from the SMHS and SWHS, two ongoing population-based cohort studies. Details of these studies have been previously described $[23,24]$. Briefly, Shanghai residents aged 40-70 years were recruited to the SWHS from 1996-2000 and aged 40-74 years to the SMHS from 2002-2006. Information on demographic/lifestyle factors was collected at baseline through in-person interviews via structured questionnaires by trained interviewers. Cancer occurrence information was collected via annual record linkage with the population-based Shanghai Cancer Registry supplemented by in-person surveys every 2-4 years. Matches were verified by home visits. Cancer diagnoses were verified and clinical information was extracted through review of medical charts obtained from the diagnosing hospital.

Participants with a first cancer diagnosis of colorectal cancer (International Classification of Diseases ninth edition [ICD-9] codes 153 and 154), lung cancer (ICD-9 code 162), gastric cancer (ICD-9 code 151), and breast cancer (among females only) (ICD-9 code 174) [25,26] after study enrollment were selected for this study and were sent for linkage to EMR $(n=4,436)$. Diabetes medication use from 2004-2014 was 
extracted from EMR for 3,438 participants. We excluded participants diagnosed with cancer prior to 2004 due to incompleteness of EMR data collection of medication use prior to this time point $(n=548)$, resulting in 2,890 cancer cases for the analysis.

\section{Ascertainment of diabetes diagnosis and medication use}

Information on diagnosis of other chronic diseases, including diabetes, was obtained through active surveys conducted every 2-4 years in both cohorts. In 2015, we linked cancer cases identified from the SWHS and SMHS to EMR data from the Changning District Health Information Center to obtain common diseases and medication use information. The data included clinic visits, laboratory tests, diagnosis, hospital discharge summary, and prescriptions. Using this data, participants were categorized by diabetes medication class (metformin, sulfonylureas, insulin, glucosidase inhibitors, thiazolidinone compounds, and Chinese hypoglycemic medicine), and whether use was initiated before and/or after cancer diagnosis. In the current study, we treat patients with either self-reported diabetes history or EMR evidence of diabetes as diabetic cases.

\section{Statistical analysis}

The study endpoints were death from any cause (for overall survival analyses), and cancer-specific death from breast, colorectal, lung, or gastric cancer (for relevant cancer-specific survival analyses). The censor date for event-free individuals was the date of last in-person contact or December 31, 2014 (6 months prior to the date of the most recent record linkage), whichever was most recent. For cancer-specific mortality analysis, follow-up time for patients who died of other causes was censored.

Demographic/lifestyle and clinical/treatment factors were tabulated by cancer type. The 3- and 5-year survival rates were calculated using the life table analysis method and compared using the log-rank test; $34.3 \%$ of participants had $\geq 5$ years of survival time (from date of diagnosis to death or date of last follow-up). Cox proportional hazards models with age as the time scale starting at age of cancer diagnosis were used to estimate the associations of survival outcomes with use of diabetes medications of interest, including metformin, sulfonylureas, and insulin [27]. Hazard ratio (HR) refers to use versus non-use of specific diabetes medications. The proportional hazards assumptions were evaluated by checking with the Schoenfeld residuals. Stratified Cox proportional hazards models were used for some categorical covariates that did not satisfy the proportional assumption. To alleviate the concern of the immortal time bias for medication uses initiated after cancer diagnoses, those medica- tion uses were treated as the time-dependent variables in the survival analysis [28]. The covariates adjusted for in the Cox models were sex, education, body mass index (BMI), cigarette smoking status, regular exercise, comorbidity (i.e., myocardial infarction, congestive heart failure, peripheral vascular disease, cerebrovascular disease), TNM stages of cancer, and cancer treatment (surgery, chemotherapy, and radiotherapy). Use of diabetes medications were mutually adjusted in the analysis. The analyses were conducted for each type of cancer separately and for all four types of cancer combined. Two sets of analyses were carried out: model 1analysis included all participants of the study, and model 2analysis included only diabetic patients. All statistical analyses were performed using SAS ver. 9.4 (SAS Institute, Cary, $\mathrm{NC})$.

\section{Ethical statement}

All participants provided written informed consent and the study was approved by the Institutional Review Boards (IRBs) at all participating institutions.

\section{Results}

Included in the current analysis are 2,890 cancer cases: 633 breast, 890 colorectal, 824 lung, and 543 gastric. Patient characteristics by diabetes and diabetes medication use are presented in S1 Table. Over a median follow-up of 3.4 years after cancer diagnosis (interquartile range, 1.0 to 6.3 years), 1,528 deaths were documented among 2,890 patients. The 5-year survival rate by cancer type was $87.6 \%$ (breast), $58.4 \%$ (colorectal), $18.3 \%$ (lung), and 36.7\% (gastric). Overall survival rate by cancer type was positively associated with education and surgery, but inversely associated with age at cancer diagnosis and TNM stage (Table 1). Among breast cancer cases, overall survival rate was inversely associated with baseline (pre-cancer diagnosis) BMI, current/former smoking status, and existing comorbidity. For colorectal cancer cases, overall survival rate was higher among those with chemotherapy, but lower among those with high baseline BMI, existing comorbidity and radiotherapy. For lung cancer cases, overall survival rate was higher among females, or those with no comorbidity and chemotherapy, but lower for current/former smokers. Among gastric cancer cases, overall survival rate was positively associated with chemotherapy (Table 1).

Diabetes status was associated with increased risk of overall and cancer-specific mortality among breast cancer cases (HR, 1.56; 95\% confidence interval [CI], 1.01 to 2.43 , and HR, 






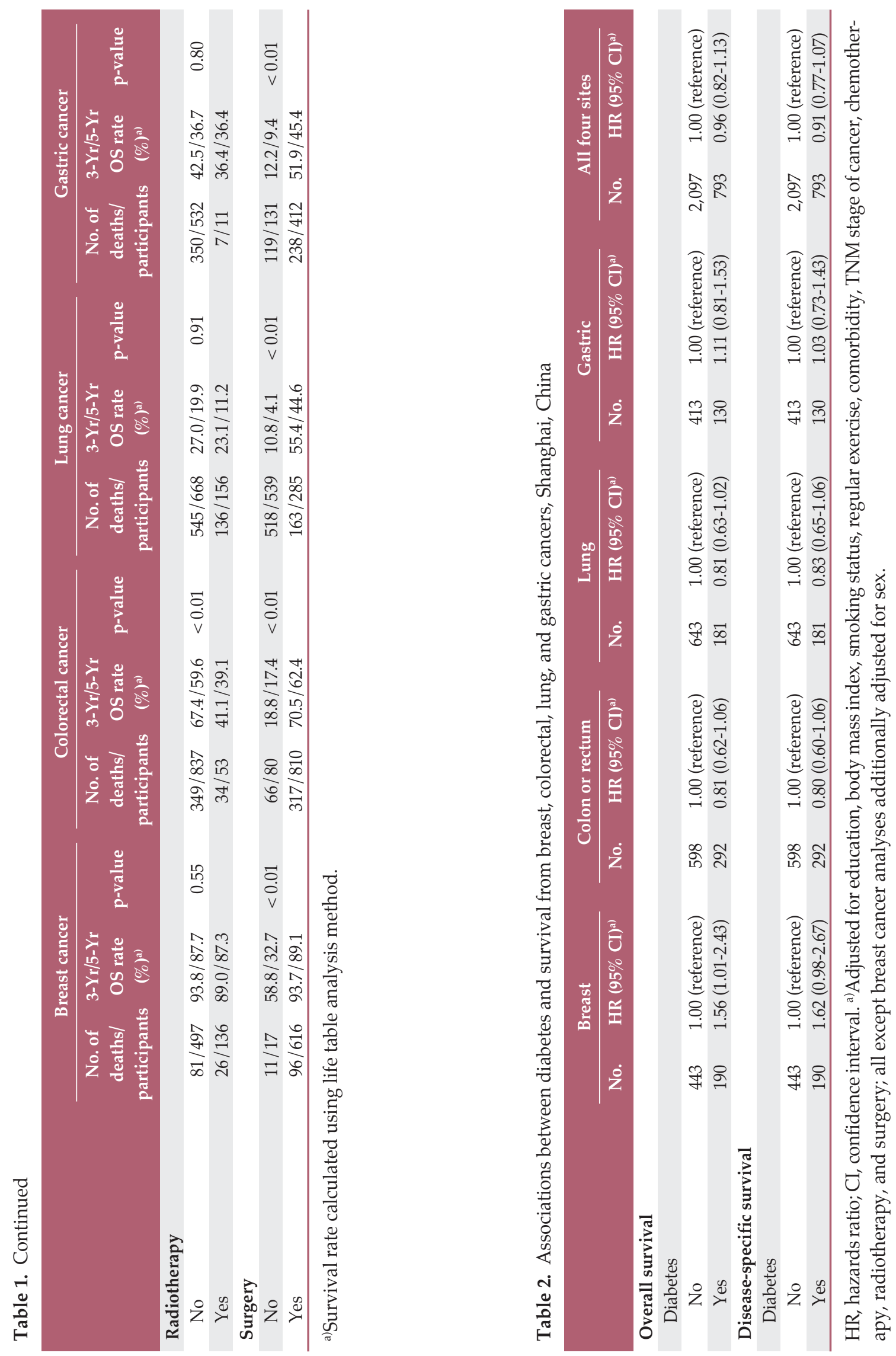
Table 3. Associations between use of diabetes medications (metformin, sulfonylureas, and insulin) and overall survival, Shanghai, China

\begin{tabular}{|c|c|c|c|c|c|}
\hline & \multicolumn{5}{|c|}{ Cancer site } \\
\hline & All 4 sites combined & Breast & Colorectal & Lung & Gastric \\
\hline \multicolumn{6}{|c|}{ Model I (all participants) } \\
\hline Events / Total & $1,528 / 2,890$ & $107 / 633$ & $383 / 890$ & $681 / 824$ & $357 / 543$ \\
\hline \multicolumn{6}{|c|}{ Use of metformin } \\
\hline Never use & 1.00 (ref.) & 1.00 (ref.) & 1.00 (ref.) & 1.00 (ref.) & 1.00 (ref.) \\
\hline Ever use & $0.75(0.57-0.98)$ & $0.73(0.35-1.53)$ & $0.55(0.34-0.88)$ & $0.93(0.61-1.40)$ & $0.95(0.52-1.74)$ \\
\hline \multicolumn{6}{|c|}{ Use of sulfonylureas } \\
\hline Never use & 1.00 (ref.) & 1.00 (ref.) & 1.00 (ref.) & 1.00 (ref.) & 1.00 (ref.) \\
\hline Ever use & $1.03(0.82-1.30)$ & $1.65(0.87-3.10)$ & $1.02(0.69-1.52)$ & $0.79(0.55-1.12)$ & $1.00(0.60-1.68)$ \\
\hline \multicolumn{6}{|l|}{ Use of insulin } \\
\hline Never use & 1.00 (ref.) & 1.00 (ref.) & 1.00 (ref.) & 1.00 (ref.) & 1.00 (ref.) \\
\hline Ever use & $1.89(1.57-2.29)$ & $1.94(1.05-3.56)$ & $2.60(1.90-3.54)$ & $1.43(1.06-1.94)$ & $1.45(0.99-2.10)$ \\
\hline \multicolumn{6}{|c|}{ Model II (all diabetic patients) } \\
\hline Events/Total & $373 / 793$ & $38 / 190$ & $116 / 292$ & $138 / 181$ & $81 / 130$ \\
\hline \multicolumn{6}{|c|}{ Use of metformin } \\
\hline Never use & 1.00 (ref.) & 1.00 (ref.) & 1.00 (ref.) & 1.00 (ref.) & 1.00 (ref.) \\
\hline Ever use & $0.74(0.53-1.02)$ & $0.76(0.33-1.75)$ & $0.51(0.30-0.88)$ & $1.56(0.87-2.79)$ & $1.01(0.48-2.12)$ \\
\hline \multicolumn{6}{|c|}{ Use of sulfonylureas } \\
\hline Never use & 1.00 (ref.) & 1.00 (ref.) & 1.00 (ref.) & 1.00 (ref.) & 1.00 (ref.) \\
\hline Ever use & $1.49(1.09-2.03)$ & $2.87(1.22-6.80)$ & $1.50(0.93-2.41)$ & $1.03(0.62-1.71)$ & $2.05(1.09-3.84)$ \\
\hline \multicolumn{6}{|l|}{ Use of insulin } \\
\hline Never use & 1.00 (ref.) & 1.00 (ref.) & 1.00 (ref.) & 1.00 (ref.) & 1.00 (ref.) \\
\hline Ever use & $1.70(1.26-2.29)$ & $1.36(0.64-2.89)$ & $4.06(2.59-6.39)$ & $1.15(0.70-1.88)$ & $1.36(0.69-2.69)$ \\
\hline
\end{tabular}

Hazard ratios adjusted for education, body mass index, smoking status, regular exercise, comorbidity, TNM stage of cancer, chemotherapy, radiotherapy, and surgery; all except breast cancer analyses additionally adjusted for sex. During analyses, use of diabetes medications (metformin, sulfonylureas, and insulin) was also adjusted mutually in the models.

$1.62 ; 95 \% \mathrm{CI}, 0.98$ to 2.67 , respectively) after adjustment for patient and clinical characteristics. However, no such associations were detected in colorectal, lung, and gastric cancer cases (Table 2).

Among all participants (diabetics and non-diabetics) in the study, better overall survival was found among metformin users with colorectal cancer (HR, $0.55 ; 95 \% \mathrm{CI}, 0.34$ to 0.88 ) or when all four cancer types were combined (HR, 0.75; $95 \%$ CI, 0.57 to 0.98 ) (Table 3) compared to non-users. Ever use of insulin among all participants was associated with worse survival when all four cancer types were combined (HR, 1.89; 95\% CI, 1.57 to 2.29) and individually for breast (HR, 1.94; 95\% CI, 1.05 to 3.56), colorectal (HR, 2.60; 95\% CI, 1.90 to $3.54)$, lung (HR, 1.43; 95\% CI, 1.06 to 1.94), and gastric cancer (HR, 1.45; 95\% CI, 0.99 to 2.10) (Table 3). When the analysis was restricted to diabetic patients only, the association of sulfonylureas use with worse overall survival was observed for breast or gastric cancer (HR, 2.87; 95\% CI, 1.22 to 6.80 and HR, 2.05; $95 \%$ CI, 1.09 to 3.84, respectively) and for all four cancer sites combined (HR, 1.49; 95\% CI, 1.09 to 2.03). The association of metformin use with better overall survival and the association of insulin use with worse overall survival were also evident in colorectal cancer and all four cancer types combined when the analysis was restricted to diabetics (Table 3).

Similar to the observed associations between diabetes medication use and overall survival, metformin use was associated with better cancer-specific survival, while ever use of insulin was associated with worse cancer-specific survival in colorectal cancer and all four cancer types combined among all participants or diabetic patients only (Table 4). An association between use of sulfonylureas with worse cancerspecific survival was also observed for all four cancer types combined (HR, 1.45; 95\% CI, 1.08 to 1.94) among diabetics (Table 4). 
Table 4. Associations between use of diabetes medications (metformin, sulfonylureas, and insulin) and cancer-specific survival, Shanghai, China

\begin{tabular}{|c|c|c|c|c|c|}
\hline & \multicolumn{5}{|c|}{ Cancer site } \\
\hline & All 4 sites combined & Breast & Colorectal & Lung & Gastric \\
\hline \multicolumn{6}{|c|}{ Model I (all participants) } \\
\hline Events/Total & $1,408 / 2,890$ & $84 / 633$ & $342 / 890$ & $648 / 824$ & $334 / 543$ \\
\hline \multicolumn{6}{|c|}{ Use of metformin } \\
\hline Never use & 1.00 (ref.) & 1.00 (ref.) & 1.00 (ref.) & 1.00 (ref.) & 1.00 (ref.) \\
\hline Ever use & $0.75(0.56-1.01)$ & $0.70(0.29-1.70)$ & $0.58(0.35-0.95)$ & $0.98(0.64-1.49)$ & $0.90(0.48-1.70)$ \\
\hline \multicolumn{6}{|c|}{ Use of sulfonylureas } \\
\hline Never use & 1.00 (ref.) & 1.00 (ref.) & 1.00 (ref.) & 1.00 (ref.) & 1.00 (ref.) \\
\hline Ever use & $0.97(0.76-1.23)$ & $1.78(0.86-3.71)$ & $1.00(0.66-1.52)$ & $0.75(0.52-1.08)$ & $0.91(0.52-1.58)$ \\
\hline \multicolumn{6}{|l|}{ Use of insulin } \\
\hline Never use & 1.00 (ref.) & 1.00 (ref.) & 1.00 (ref.) & 1.00 (ref.) & 1.00 (ref.) \\
\hline Ever use & $1.80(1.48-2.21)$ & $1.32(0.62-2.82)$ & $2.52(1.81-3.51)$ & $1.40(1.02-1.91)$ & $1.44(0.98-2.14)$ \\
\hline \multicolumn{6}{|c|}{ Model II (all diabetic patients) } \\
\hline Events/Total & $331 / 793$ & $29 / 190$ & $101 / 292$ & $128 / 181$ & $73 / 130$ \\
\hline \multicolumn{6}{|c|}{ Use of metformin } \\
\hline Never use & 1.00 (ref.) & 1.00 (ref.) & 1.00 (ref.) & 1.00 (ref.) & 1.00 (ref.) \\
\hline Ever use & $0.74(0.54-1.01)$ & $0.84(0.32-2.20)$ & $0.53(0.29-0.96)$ & $1.57(0.86-2.56)$ & $1.01(0.46-2.22)$ \\
\hline \multicolumn{6}{|c|}{ Use of sulfonylureas } \\
\hline Never use & 1.00 (ref.) & 1.00 (ref.) & 1.00 (ref.) & 1.00 (ref.) & 1.00 (ref.) \\
\hline Ever use & $1.45(1.08-1.94)$ & $2.20(0.83-5.84)$ & $1.59(0.94-2.70)$ & $0.99(0.58-1.67)$ & $1.76(0.90-3.42)$ \\
\hline \multicolumn{6}{|l|}{ Use of insulin } \\
\hline Never use & 1.00 (ref.) & 1.00 (ref.) & 1.00 (ref.) & 1.00 (ref.) & 1.00 (ref.) \\
\hline Ever use & $1.67(1.27-2.19)$ & $0.92(0.36-2.31)$ & $3.88(2.32-6.47)$ & $1.19(0.72-1.97)$ & $1.41(0.68-2.92)$ \\
\hline
\end{tabular}

Hazard ratios adjusted for education, body mass index, smoking status, regular exercise, comorbidity, TNM stage of cancer, chemotherapy, radiotherapy, and surgery; all except breast cancer analyses additionally adjusted for sex. During analyses, use of diabetes medications (metformin, sulfonylureas, and insulin) was also adjusted mutually in the models.

\section{Discussion}

Metformin, a biguanide, is the most commonly used type 2 diabetes drug [29]. It improves insulin resistance by decreasing circulating levels of glucose and insulin, which may indirectly affect cancer growth $[12,30]$. In vivo studies have shown that mice on a control diet exhibited less antitumor activity associated with metformin compared with mice on a high energy diet, suggesting that the antitumor activity of metformin may be due to reduced endogenous insulin levels [31-33]. Metformin may also have direct anti-cancer effects, including mammalian target of rapamycin inhibition through the activation of AMP-kinase, which reduces cell growth and proliferation by slowing protein synthesis $[12,34,35]$. In our study, we found metformin use was associated with decreased risk of mortality among colorectal cancer patients but not in patients with breast, lung, or gastric cancers. Our finding suggests that effects of metformin use vary by cancer type; however, underlying reasons are unclear. Studies have shown that hyperinsulinemia patients with tumor expression of the insulin receptor, LKB1, and TSC2 may respond to metformin therapy while patients without these characteristics may not [34]. More studies are needed to better understand the effects of metformin therapy in different patient populations and to identify biomarkers that may predict responsiveness to metformin.

Unlike metformin, sulfonylureas and exogenous insulin increase circulating levels of insulin [36]. Insulin is known to have mitogenic properties, and increased levels of insulin may promote carcinogenesis due to insulin's growth-promoting properties [37-39]. Previous studies have observed that patients with type 2 diabetes who were exposed to sulfonylureas and exogenous insulin had a significantly increased risk of cancer-related mortality compared with patients exposed to metformin [37]. In our study, we observed worse survival for all four cancer types among those patients who had ever used insulin, as well as for certain types of cancer, 
such as breast cancer and gastric cancer, among diabetic patients who took sulfonylureas. It is uncertain whether this increased risk is related to a deleterious effect of sulfonylurea and insulin or a protective effect of metformin or due to some unmeasured effect related to both choice of therapy and cancer risk. In addition, diabetic cases who use insulin are generally more severe cases than those who are only prescribed oral diabetes medications. This may also contribute to the higher mortality noted in insulin users.

Many diabetic patients in our study were taking multiple diabetes medications concurrently. Although we have mutually adjusted diabetes medication use in our analysis, we were unable to evaluate the association for monotherapy patients and had to rely on any use of the medication as the study variable due to the low number of monotherapy patients in our study population. We were unable to accurately account for length of medication use, due to the inability to capture use of medications prior to establishment of EMR. Studies that assess pharmacological factors, such as concurrent versus monotherapy medication use and dosage/ duration of medication use, may better assess the association between cancer survival and diabetes medication use and potential survival bias. Finally, we were missing TNM stage information for $24 \%$ of patients included in the analyses, which may have introduced a residual confounding effect.

Our study has several noteworthy strengths. The data come from large population-based studies with comprehensive data collection. Self-reported diabetes information was supplemented by EMR data, and medication use was gathered from EMR, strengthening the validity of our data. Our study evaluated survival after cancer diagnosis, rather than mortality, allowing us to evaluate the potential survival advantage of diabetes medication use among cancer patients.
Unlike most previous studies, in this study we comprehensively adjusted for a wide range of potential confounding factors. Importantly, we used time-dependent Cox proportional hazards models in the survival analysis, thus alleviating any concern of immortal time bias for medication use initiated after cancer diagnoses.

In summary, we found that use of metformin was associated with improved overall and cancer-specific survival among colorectal cancer patients. In contrast, insulin use was associated with worse survival from breast, lung, colorectal, and gastric cancers. Sulfonylurea use was associated with worse survival among breast or gastric cancer patients with diabetes. Additional investigation on the topic is needed given the potential translational impact if our findings are proven to be true.

\section{Electronic Supplementary Material}

Supplementary materials are available at Cancer Research and Treatment website (https: // www.e-crt.org).

\section{Conflicts of Interest}

Conflict of interest relevant to this article was not reported.

\section{Acknowledgments}

This work was supported by NIH grants for the SMHS [UM1 CA173640]; SWHS [UM1 CA182910] studies; State Key Project Specialized for Infectious Diseases China [2008ZX10002-015, 2012ZX10002008-002]; and National Center for Advancing Translational Sciences [TL1TR000447 to M.L.B].

\section{References}

1. Barone BB, Yeh HC, Snyder CF, Peairs KS, Stein KB, Derr RL, et al. Long-term all-cause mortality in cancer patients with preexisting diabetes mellitus: a systematic review and metaanalysis. JAMA. 2008;300:2754-64.

2. Noto H, Goto A, Tsujimoto T, Noda M. Cancer risk in diabetic patients treated with metformin: a systematic review and meta-analysis. PLoS One. 2012;7:e33411.

3. Decensi A, Puntoni M, Goodwin P, Cazzaniga M, Gennari A, Bonanni B, et al. Metformin and cancer risk in diabetic patients: a systematic review and meta-analysis. Cancer Prev Res (Phila). 2010;3:1451-61.

4. Zhang P, Li H, Tan X, Chen L, Wang S. Association of metformin use with cancer incidence and mortality: a meta-analysis. Cancer Epidemiol. 2013;37:207-18.
5. Baur DM, Klotsche J, Hamnvik OP, Sievers C, Pieper L, Wittchen HU, et al. Type 2 diabetes mellitus and medications for type 2 diabetes mellitus are associated with risk for and mortality from cancer in a German primary care cohort. Metabolism. 2011;60:1363-71.

6. Bo S, Ciccone G, Rosato R, Villois P, Appendino G, Ghigo E, et al. Cancer mortality reduction and metformin: a retrospective cohort study in type 2 diabetic patients. Diabetes Obes Metab. 2012;14:23-9.

7. Landman GW, Kleefstra N, van Hateren KJ, Groenier KH, Gans RO, Bilo HJ. Metformin associated with lower cancer mortality in type 2 diabetes: ZODIAC-16. Diabetes Care. 2010;33:322-6.

8. Currie CJ, Poole CD, Jenkins-Jones S, Gale EA, Johnson JA, 
Morgan CL. Mortality after incident cancer in people with and without type 2 diabetes: impact of metformin on survival. Diabetes Care. 2012;35:299-304.

9. He X, Esteva FJ, Ensor J, Hortobagyi GN, Lee MH, Yeung SC. Metformin and thiazolidinediones are associated with improved breast cancer-specific survival of diabetic women with HER2+ breast cancer. Ann Oncol. 2012;23:1771-80.

10. Bayraktar S, Hernadez-Aya LF, Lei X, Meric-Bernstam F, Litton JK, Hsu L, et al. Effect of metformin on survival outcomes in diabetic patients with triple receptor-negative breast cancer. Cancer. 2012;118:1202-11.

11. Lee JH, Kim TI, Jeon SM, Hong SP, Cheon JH, Kim WH. The effects of metformin on the survival of colorectal cancer patients with diabetes mellitus. Int J Cancer. 2012;131:752-9.

12. Ahmed I, Ferro A, Cohler A, Langenfeld J, Surakanti SG, Aisner J, et al. Impact of metformin use on survival in locallyadvanced, inoperable non-small cell lung cancer treated with definitive chemoradiation. J Thorac Dis. 2015;7:346-55.

13. Lin J, Gill A, Zahm SH, Carter CA, Shriver CD, Nations JA, et al. Metformin use and survival after non-small cell lung cancer: a cohort study in the US Military health system. Int J Cancer. 2017;141:254-63.

14. Ramjeesingh R, Orr C, Bricks CS, Hopman WM, Hammad N. A retrospective study on the role of diabetes and metformin in colorectal cancer disease survival. Curr Oncol. 2016;23:e11622.

15. Paulus JK, Williams CD, Cossor FI, Kelley MJ, Martell RE. Metformin, diabetes, and survival among U.S. Veterans with colorectal cancer. Cancer Epidemiol Biomarkers Prev. 2016; 25:1418-25.

16. Lee CK, Jung M, Jung I, Heo SJ, Jeong YH, An JY, et al. Cumulative metformin use and its impact on survival in gastric cancer patients after gastrectomy. Ann Surg. 2016;263:96-102.

17. Lacroix O, Couttenier A, Vaes E, Cardwell CR, De Schutter H, Robert A. Impact of metformin on gastric adenocarcinoma survival: a Belgian population based study. Cancer Epidemiol. 2018;53:149-55.

18. Ioacara S, Guja C, Ionescu-Tirgoviste C, Fica S, Roden M. Cancer specific mortality in insulin-treated type 2 diabetes patients. PLoS One. 2014;9:e93132.

19. Bowker SL, Yasui Y, Veugelers P, Johnson JA. Glucose-lowering agents and cancer mortality rates in type 2 diabetes: assessing effects of time-varying exposure. Diabetologia. 2010;53:1631-7.

20. Suissa S. Immortal time bias in observational studies of drug effects. Pharmacoepidemiol Drug Saf. 2007;16:241-9.

21. Chaiteerakij R, Petersen GM, Bamlet WR, Chaffee KG, Zhen DB, Burch PA, et al. Metformin use and survival of patients with pancreatic cancer: a cautionary lesson. J Clin Oncol. 2016;34:1898-904.

22. Xin W, Fang L, Fang Q, Zheng X, Huang P. Effects of metformin on survival outcomes of pancreatic cancer patients with diabetes: a meta-analysis. Mol Clin Oncol. 2018;8:483-8.
23. Lee SA, Xu WH, Zheng W, Li H, Yang G, Xiang YB, et al. Physical activity patterns and their correlates among Chinese men in Shanghai. Med Sci Sports Exerc. 2007;39:1700-7.

24. Zheng W, Chow WH, Yang G, Jin F, Rothman N, Blair A, et al. The Shanghai Women's Health Study: rationale, study design, and baseline characteristics. Am J Epidemiol. 2005;162: 1123-31.

25. Centers for Disease Control and Prevention [Internet]. Atlanta, GA: Centers for Disease Control and Prevention; 2018 [cited 2018 Jun 1]. Available from: https:// www.cdc.gov/nhsn/xls / icd-10-cm-ddc.xlsx I--CDDC.

26. ICD10Data.com [Internet]. ICD10Data.com; 2018 [cited 2018 Jun 1]. Available from: http://www.icd10data.com/ICD10CM /Codes/E00-E89/E08-E13/E11- I--CDCETd.

27. Korn EL, Graubard BI, Midthune D. Time-to-event analysis of longitudinal follow-up of a survey: choice of the time-scale. Am J Epidemiol. 1997;145:72-80.

28. Hanley JA, Foster BJ. Avoiding blunders involving 'immortal time'. Int J Epidemiol. 2014;43:949-61.

29. Belda-Iniesta C, Pernia O, Simo R. Metformin: a new option in cancer treatment. Clin Transl Oncol. 2011;13:363-7.

30. Howlett HC. Metformin in the management of type 2 diabetes. Diabet Med. 1989;6:179-80.

31. Algire C, Zakikhani M, Blouin MJ, Shuai JH, Pollak M. Metformin attenuates the stimulatory effect of a high-energy diet on in vivo LLC1 carcinoma growth. Endocr Relat Cancer. 2008;15:833-9.

32. Belfiore A, Frasca F, Pandini G, Sciacca L, Vigneri R. Insulin receptor isoforms and insulin receptor/insulin-like growth factor receptor hybrids in physiology and disease. Endocr Rev. 2009;30:586-623.

33. Cao X, Wen ZS, Wang XD, Li Y, Liu KY, Wang X. The clinical effect of metformin on the survival of lung cancer patients with diabetes: a comprehensive systematic review and metaanalysis of retrospective studies. J Cancer. 2017;8:2532-41.

34. Dowling RJ, Goodwin PJ, Stambolic V. Understanding the benefit of metformin use in cancer treatment. BMC Med. 2011;9:33.

35. Gotlieb WH, Saumet J, Beauchamp MC, Gu J, Lau S, Pollak $\mathrm{MN}$, et al. In vitro metformin anti-neoplastic activity in epithelial ovarian cancer. Gynecol Oncol. 2008;110:246-50.

36. Bowker SL, Majumdar SR, Veugelers P, Johnson JA. Increased cancer-related mortality for patients with type 2 diabetes who use sulfonylureas or insulin. Diabetes Care. 2006;29:254-8.

37. Barker BE, Fanger H, Farnes P. Human mammary slices in organ xulture. I. Method of culture and preliminary observations on the effect of insulin. Exp Cell Res. 1964;35:437-48.

38. Rosenfeld RG. Insulin-like growth factors and the basis of growth. N Engl J Med. 2003;349:2184-6.

39. Del Giudice ME, Fantus IG, Ezzat S, McKeown-Eyssen G, Page D, Goodwin PJ. Insulin and related factors in premenopausal breast cancer risk. Breast Cancer Res Treat. 1998;47:111-20. 